\title{
Identification of the molecular signatures integral to regenerating photoreceptors in the retina of the zebra fish
}

\author{
Sonya E. L. Craig • Anda-Alexandra Calinescu • \\ Peter F. Hitchcock
}

Received: 21 May 2008/Accepted: 26 August 2008/Published online: 18 November 2008

(C) The Author(s) 2008. This article is published with open access at Springerlink.com

\begin{abstract}
Investigating neuronal and photoreceptor regeneration in the retina of zebra fish has begun to yield insights into both the cellular and molecular means by which this lower vertebrate is able to repair its central nervous system. However, knowledge about the signaling molecules in the local microenvironment of a retinal injury and the transcriptional events they activate during neuronal death and regeneration is still lacking. To identify genes involved in photoreceptor regeneration, we combined light-induced photoreceptor lesions, laser-capture microdissection of the outer nuclear layer (ONL) and analysis of gene expression to characterize transcriptional changes for cells in the ONL as photoreceptors die and are regenerated. Using this approach, we were able to characterize aspects of the molecular signature of injured and dying photoreceptors, cone photoreceptor progenitors, and microglia within the ONL. We validated changes in gene expression and characterized the cellular expression for three novel, extracellular signaling molecules that we hypothesize are involved in regulating regenerative events in the retina.
\end{abstract}

Electronic Supplementary Material The online version of this article (doi:10.1007/s12177-008-9011-5) contains supplementary material, which is available to authorized users.

S. E. L. Craig · A.-A. Calinescu • P. F. Hitchcock $(\triangle)$ Department of Ophthalmology and Visual Sciences,

Kellogg Eye Center, University of Michigan Medical School, 1000 Wall Street,

Ann Arbor, MI 48105, USA

e-mail: peterh@med.umich.edu

S. E. L. Craig · A.-A. Calinescu • P. F. Hitchcock

Cell and Developmental Biology,

The Neuroscience Graduate Program,

University of Michigan Medical School,

1000 Wall Street,

Ann Arbor, MI 48105, USA
Keywords Regenerative neurogenesis $\cdot$ Retinal stem cells · Laser-capture microdissection · Microarray · Microglia

\section{Introduction}

In the teleost retina, extensive neuronal death $[20,30]$ or the selective death of photoreceptors $[35,38]$ stimulates intrinsic stem cells to proliferate and give rise to regenerated neurons, which then integrate into existing neural circuits (see [18]). Consequently, the teleost retina is an ideal system to study the intrinsic cellular and molecular mechanisms that allow stem cells to regenerate neurons in the vertebrate central nervous system (CNS). Identifying and testing the molecular determinants of neuronal regeneration in the teleost retina should yield results that will have implications for understanding the regenerative potential of stem cells in the human retina and brain and the potential use of stem-cell-based therapies to treat CNS injuries or disease.

Significant progress has been made in characterizing the cells that are involved in mediating neuronal regeneration of the teleost retina. Injury to photoreceptors was long considered necessary to elicit regeneration [30], although a recent study showed that cell death within the inner nuclear layer (INL) that spares photoreceptors is sufficient [11]. Irrespective of the locus of cell death, Müller glia are the regenerative stem cells [3, 9], and re-expression of developmental regulatory genes and proliferation by Müller glia are required for neuronal regeneration [10, 33]. Although dying neurons and Müller glia are obligatory components of this regenerative neurogenesis, microglia resident within the retina migrate to the site of injury [31, 38] and are also likely critical for neuronal regeneration. Finally, in teleosts, photoreceptors are regenerated in their 
normal birth order, cones before rods [23, 29, 38]. Following photoreceptor-only death, injury-induced progenitors serve exclusively as "cone progenitors." Therefore, the teleost retina also provides a model in which to elucidate the molecular mechanisms that govern the selective regeneration of cones.

Since none of the cell types described above is unique to teleost fish $[12,25,26]$, a fundamental difference between animals capable of neuronal regeneration and those that are not may be the molecules elicited in response to injury. Previous studies have described the transcriptional changes that occur in the teleost retina in response to either global [6] or photoreceptor-specific injuries [21], and these studies have provided insight into the molecular basis for neuronal regeneration. Particularly revealing are changes in the molecular signature of Müller glia as they adopt the features of neural stem cells [21]. However, knowledge about signaling molecules in the local microenvironment of a retinal injury and the transcriptional events they activate during neuronal death and regeneration is still lacking.

As a means to identify genes necessary for photoreceptor regeneration, we evaluated transcriptional changes for cells in the outer nuclear layer (ONL) as photoreceptors die and are regenerated. To accomplish this, we combined lightinduced photoreceptor lesions, laser-capture microdissection (LCM) of the ONL and analysis of gene expression using oligonucleotide arrays. By selectively harvesting cells from the ONL, we were able to limit our analysis to transcriptional changes among cells within the site of injury. By this approach, we hope further to characterize the molecular signatures of injured and dying photoreceptors, cone progenitors, and activated microglia. Further, we are particularly interested in identifying novel extracellular signaling molecules involved in these regenerative events, and here we describe the cellular expression of three such factors.

\section{Materials and methods}

Animals and experimental treatments Wild-type adult zebra fish (3.0-4.0 cm long) were used in this study. Two separate paradigms were employed to induce photoreceptor death. First, for the gene array experiments and analysis of galectin-1-like 2 and galectin-9-like 1 expression, animals were housed in the dark for 7 days then exposed to fluorescent light of moderate intensity (approximately $27,000 \mathrm{~lx}$ ) for periods between 6 and $72 \mathrm{~h}$. This lesioning paradigm results in the death of photoreceptors in the dorsal retina, while sparing photoreceptors in the ventral retina (see [35, 36]). Second, for in situ hybridization for progranulin-a (see below), animals were exposed to the high-intensity light of a mercury arc lamp $(>180,000 \mathrm{~lx})$ for $30 \mathrm{~min}$. This method kills photoreceptors in a narrow horizontally oriented band across the nasal-temporal axis of the retina. To characterize gene expression in the progeny of dividing Müller glia, $\operatorname{Tg}(g f a p: G F P)^{\text {mi2001 }}$ reporter fish [2] were used. In this line, astrocytes throughout the central nervous system and Müller glia within the retina synthesize green fluorescent protein.

Protocols for all procedures using animals were approved by the University Committee for Use and Care of Animals at the University of Michigan and conform to National Institutes of Health guidelines.

Histology Fish were anesthetized in $0.1 \%$ methane sulfonate salt (MS222; Sigma Aldridge, St. Louis, MO, USA). Eyecups were then dissected, fixed overnight in $4 \%$ paraformaldehyde, cryoprotected by infiltration in $20 \%$ sucrose in phosphate buffer, and frozen in OCT (Sakura Finetek, Torrance, CA, USA). Radial sections $(8 \mu \mathrm{m})$ were cut with a cryostat and mounted on glass slides.

Immunohistochemistry was performed using standard procedures. Briefly, sections were rinsed in phosphatebuffered saline and $0.5 \%$ triton X-100 (PBST), incubated with $20 \%$ normal goat serum (NGS) in PBST, followed by overnight incubation at $4^{\circ} \mathrm{C}$ in primary antibody (anti-4C4, gift from Dr. Pamela Raymond; anti-zebra-fish galectin-1like-2, gift from Geraldo Vasta; rabbit anti-GFP, Invitrogen Corp., Carlsbad, CA, USA) at a concentration of 1:200 diluted in $2 \%$ NGS-PBST. After washing with PBST, sections were incubated in fluorescently labeled Alexafluor 555 or Alexa 488 secondary antibodies diluted 1:500 in 2\% NGS-PBST (Molecular Probes, Eugene, OR) for $1.5 \mathrm{~h}$ at room temperature, washed extensively in PBST, and sealed with mounting media and glass coverslips. Sections were counterstained with 1:1,000 dilution of bisbenzimide to label nuclei. For immunostaining with antibodies against proliferating cell nuclear antigen (PCNA; Sigma clone PC10, St. Louis, MO, USA), slides were first boiled for $20 \mathrm{~min}$ in $10 \mathrm{mM}$ sodium citrate, $\mathrm{pH} 6.0$, with $0.05 \%$ Tween 20 prior to the first rinse in PBST.

To identify dying cells in cryosections, terminal deoxynucleotide end labeling (TUNEL) with TUNEL kit (Roche Diagnostics, Indianapolis, IN, USA) was used according to the manufacturer's protocol.

In situ hybridization was performed on cryosections as previously described [19]. Briefly, sense and antisense riboprobes were synthesized from linearized plasmids, and digoxigenin (DIG)-labeled probes were generated by in vitro transcription using the DIG RNA labeling kit (Roche Diagnostics, Indianapolis, IN, USA). Following prehybridization, $200 \mathrm{ng}$ of probe in $80 \mu \mathrm{l}$ of hybridization solution was pipetted onto each slide, coverslipped, and hybridized overnight at $55^{\circ} \mathrm{C}$. The next day, the sections were washed 
and digoxigenin was immunolabeled using an alkalinephosphatase-conjugated antibody. NBT-BCIP or Fast Red (Roche Diagnostics, Indianapolis, IN, USA) served as the enzymatic substrate. After the color reaction, sections were fixed in $4 \%$ paraformaldehyde for $15 \mathrm{~min}$ before proceeding with immunohistochemistry.

The cDNA encoding galectin-9-like 1 (Accession number BC059573, Open Biosystems, Huntsville, AL, USA) was linearized with EcoRI, and riboprobes were synthesized with T7 polymerase. Similarly, the cDNA encoding progranulin-a (Accession number NM001001949; a gift from Dr. Hugh Bennett) was linearized with SmaI, and riboprobes were synthesized with T3 polymerase. Negative controls were riboprobes encoding the sense strand of the respective cDNAs, which failed to hybridize to retinal sections (data not shown).

Sections were photographed with using a Nikon E300 photomicroscope and a Nikon DMX 1200 digital camera. Images were compiled in Adobe Photoshop CS2 (Adobe, San Jose, CA, USA). Images were resized and occasionally modified for contrast and brightness using the ImageAdjustments-Contrast-Brightness setting. All images within an experiment were manipulated in exactly the same manner.

Laser-capture microdissection Eyecups were dissected from anesthetized fish and immediately frozen in OCT. Based on the timing of photoreceptor cell death and subsequent retinal stem cell proliferation (see "Results"), five time points were selected to harvest cells from the outer nuclear layers: 0 (control), 6, 12, 24, and $48 \mathrm{~h}$ after light onset. Control retinas were from fish removed from the tank and sacrificed immediately prior to turning on the lights. Care was taken throughout to avoid RNase contamination. Eyecups were stored at $-80^{\circ} \mathrm{C}$ until cryosectioning. Sections were cut at $8 \mu \mathrm{m}$, collected on silane-coated slides (Sigma, St. Louis, MO, USA), and dried in a desiccator for $45 \mathrm{~min}$. Slides were fixed and dehydrated using HistoGene frozen section staining kit (Arcturus, Molecular Devices Corporation, Sunnyvale, CA, USA) according to the manufacturer's protocol, except that $0.1 \%$ toluidine blue was used in place of the Arcturus staining reagent. Slides were air-dried in a fume hood for $15 \mathrm{~min}$ prior to LCM. An AutoPix robot (Arcuturus, Molecular Devices Corporation) was used for all microdissections. Images were captured before and after LCM using the $\times 4$ and $\times 20$ objectives on the AutoPix robot. For each eye, 12 samples were collected from 12 sections, respectively, from the dorsal ONL using the "capture multiple fields" setting. Following each session of LCM, the thermoplastic film was removed from the caps and placed in a microfuge tube. RNA was isolated using PicoPure RNA isolation kit (Arcturus, Molecular Devices Corporation) according to the manufacturer's protocol. Due
6
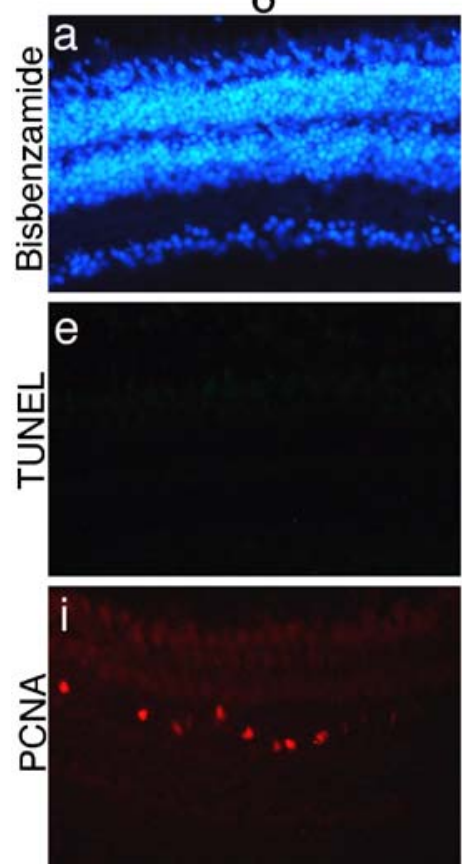

Fig. 1 Light induces photoreceptor-specific cell death and triggers proliferation. Animals were exposed to constant light and retinal sections were prepared from animals after $6 \mathrm{~h}(a, e, i), 12 \mathrm{~h}(b, f, j)$, $24 \mathrm{~h}(c, g, k)$, and $48 \mathrm{~h}(d, h, l)$. Sections were stained with bisbenzimide to label nuclear layers $(a-d)$. The same retinal sections
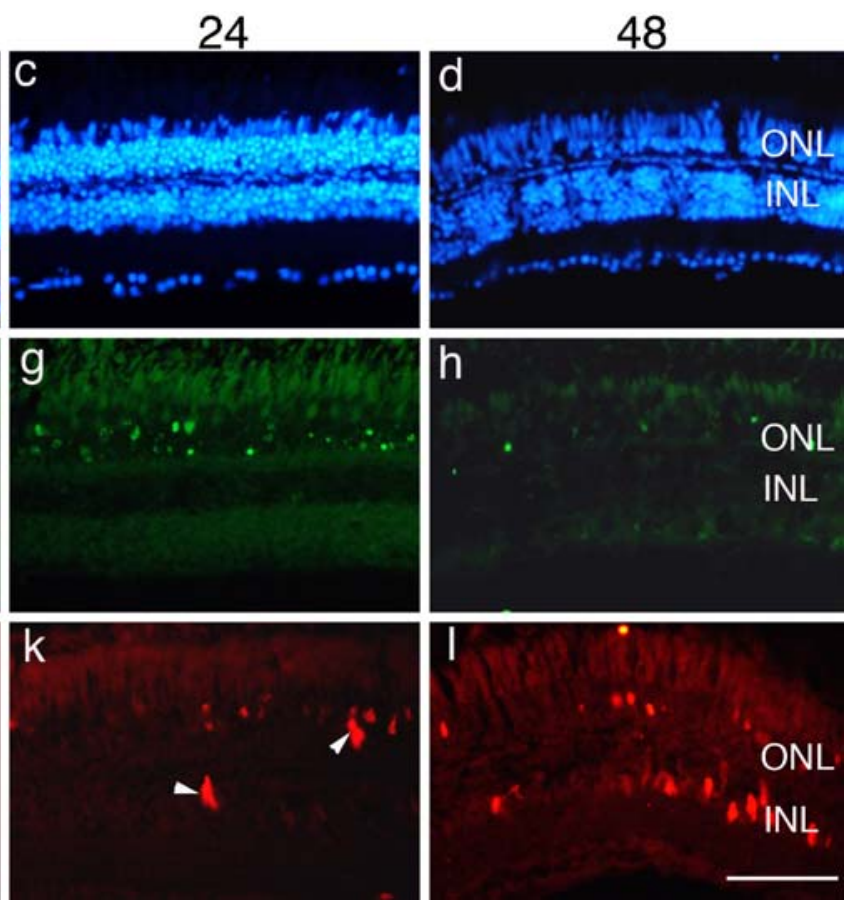

illustrated in $a-d$ were labeled with TUNEL (arrowheads, $e-h$ ). Adjacent sections were immunolabeled with antibodies against PCNA (arrowheads, $i-l$ ). Scale bar $=50 \mu \mathrm{m}$. $O N L$, outer nuclear layer; $I N L$, inner nuclear layer 

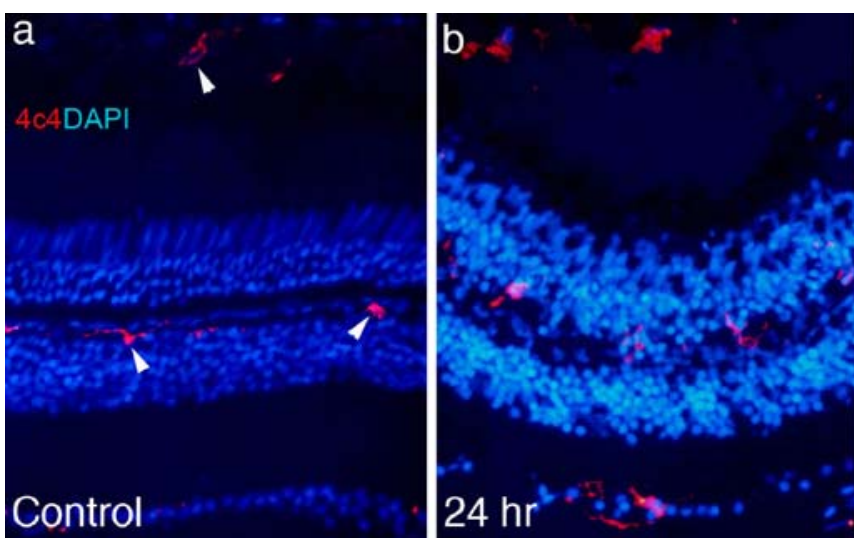

Fig. 2 As photoreceptors die, resident microglia migrate into the outer nuclear and outer segment layers. Retinal sections were taken from control (a) and experimental retinas exposed to constant light for $24 \mathrm{~h} \mathrm{(b),} 48 \mathrm{~h} \mathrm{(c),} \mathrm{and} 72 \mathrm{~h} \mathrm{(d)} \mathrm{and} \mathrm{immunolabeled} \mathrm{with} \mathrm{the} \mathrm{microglia-}$

to the length of an LCM session and the need to limit variability between sessions, one retina (12 samples) was processed per day, from cryosectioning to RNA isolation.

Microarray analysis mRNA was amplified from total RNA to yield double-stranded antisense (or complementary) RNA (aRNA) using RiboAmp HS RNA amplification kit (Arcturus, Molecular Devices Corporation). Biotinylated aRNA was generated by in vitro transcription using Affymetrix GeneChip Expression IVT Labeling Kit (Affymetrix, Santa Clara, CA, USA). Quality of the biotin-labeled aRNA was verified using Agilent Bioanalyzer nanochips. High-quality aRNA was identified as having a smooth curve and peak between 29 and $34 \mathrm{~s}$, indicating an average amplicon size of 500 bp. aRNA was quantified using Quant-iT RiboGreen RNA reagent (Molecular Probes, St. Louis, MO, USA). Five micrograms of labeled aRNA was fragmented and hybridized to Zebra fish Genome Arrays (Affymetrix, Inc., Santa Clara, CA, USA), which contain approximately 15,500 probe sets representing 14,900 genes. The hybridized arrays were scanned using a GeneChip Scanner 3000 (Affymetrix). aRNA from one retina was hybridized to a single array. For each of the five time points, three biological replicates were processed and analyzed. Each biological replicate consisted of RNA collected from a single retina (i.e., samples were not pooled).

CHP files were generated by Affymetrix Microarray Suite v5.0, prior to normalization (quantile method) with RMA software from the R project (http://www.r-project. org). False discovery rate confidence interval was used to identify statistically significant changes in gene expression between the $0 \mathrm{~h}$ control retinas and retinas exposed to light for $6,12,24$, or $48 \mathrm{~h}[1,17]$. Uncentered correlation and centroid linkage hierarchical clustering was performed using Gene Cluster 3.0, and heat maps were generated using Java TreeView 1.1.1 (http://jtreeview.sourceforge.

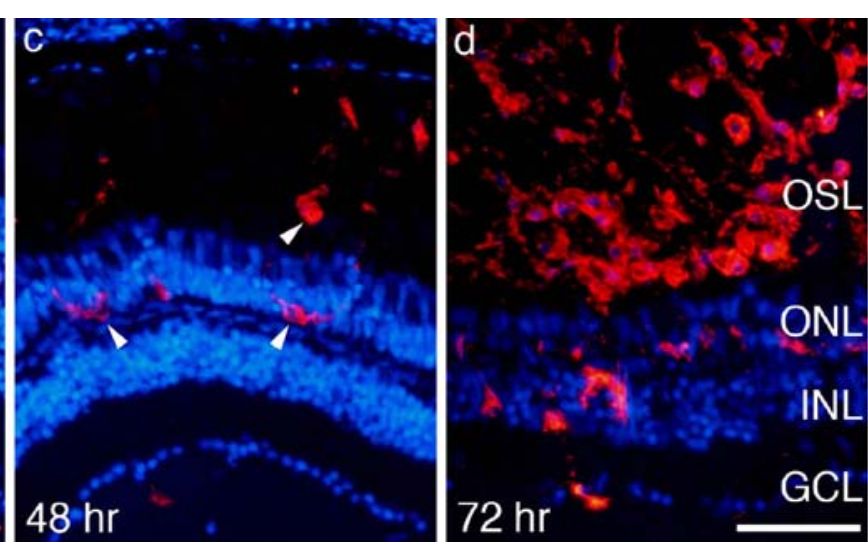

specific antibody, 4C4 (arrowheads), and counterstained with 4',6diamidino-2-phenylindole. Scale bar $=50 \mu \mathrm{m}$. OSL, outer segment layer; $O N L$, outer nuclear layer; $I N L$, inner nuclear layer; $G C L$, ganglion cell layer

net). MAIME-compliant files containing the array data are posted at the National Center for Biotechnology Information Gene expression omnibus (http://www.ncbi.nlm.nih. gov.proxy.lib.umich.edu/geo/).

\section{Results}

Histological analysis of photoreceptor degeneration in adult pigmented zebra fish

To characterize the time course of the degeneration of photoreceptors, nuclear staining with 4',6-diamidino-2phenylindole was used to evaluate the number and organization of nuclei in the ONL; photoreceptor death was evaluated with TUNEL staining, and cell proliferation was evaluated by immunostaining with antibodies against
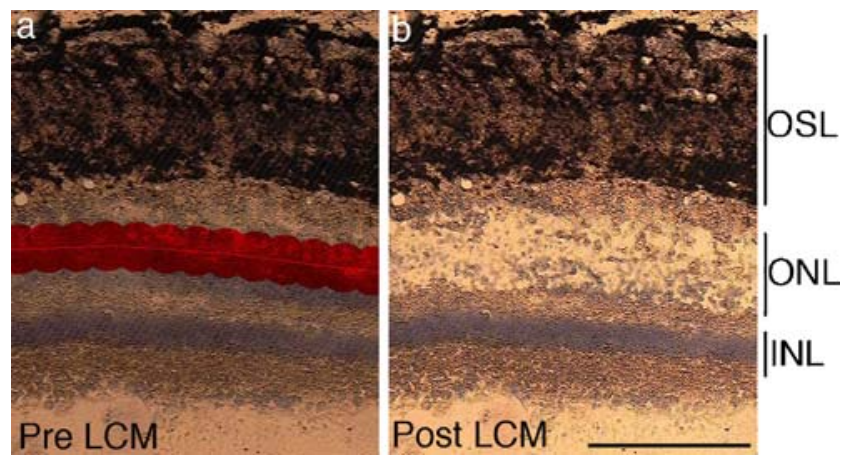

Fig. 3 Laser-capture microdissection of cells from the $O N L$. A video image of a section from a control retina is illustrated in $\mathbf{a}$ and $\mathbf{b}$. The $O N L$ is highlighted using the LCM robot, identifying the area selected for dissection (a). Panel b illustrates the retinal section in a after LCM. Scale bar $=100 \mu \mathrm{m}$. OSL, outer segment layer; $O N L$, outer nuclear layer; $I N L$, inner nuclear layer 
Fig. 4 Heat map representing the hierarchical clustering of 1,937 genes observed to change in expression in the ONL over the course of photoreceptor injury and regeneration. Uncentered Pearson's correlation and centroid linkage of genes were used to cluster transcriptional changes. Three clustering differences were observed: genes with expression that increased, genes with expression that decreased, and genes that showed differential expression at the 12-h time point

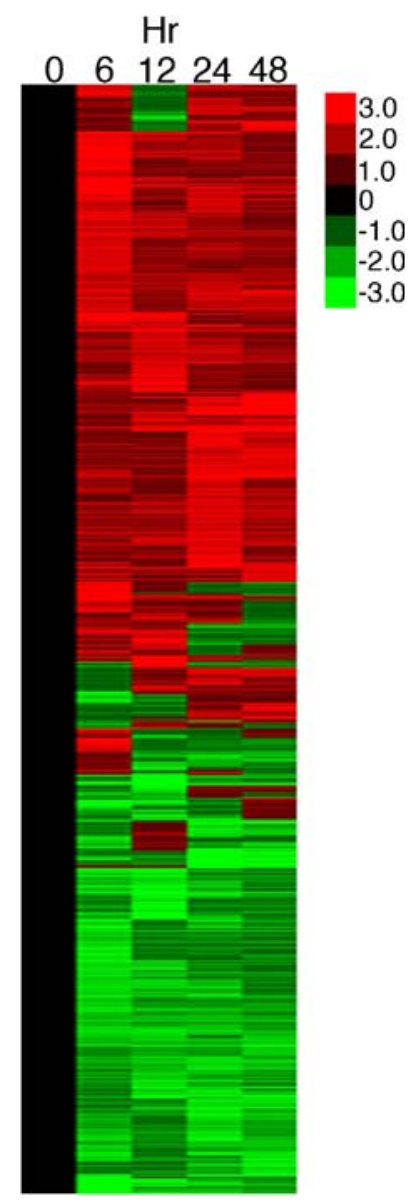

PCNA. After 6-, 12-, and 24-h exposure to moderate light, the three nuclear layers are unremarkable in appearance and little apparent injury occurs to the photoreceptors (Fig. 1ac). In contrast, $48 \mathrm{~h}$ after light onset, the ONL is markedly thinner than normal, and there is some disorganization within the inner nuclear layer (Fig. 1d). TUNEL-positive cells are present in the ONL between 12 and $48 \mathrm{~h}$ after light onset (Fig. 1f), with a peak in the number of labeled cells at $24 \mathrm{~h}$ after light onset (Fig. 1g). TUNEL-positive cells were present only in the ONL, confirming that cell death was limited to photoreceptors.

Injury-induced proliferation is first observed at $6 \mathrm{~h}$ after light onset and was restricted to cells within the ONL (Fig. 1i). We infer these are rod precursors, mitotically active cells that reside in the ONL. In contrast, at $24 \mathrm{~h}$ after light onset, dividing cells are present in the INL (Fig. 1k). These are Müller glia, which have reentered the cell cycle. By $48 \mathrm{~h}$ after light onset, clusters of injury-induced progenitors envelope the Müller glia and span the thickness of the INL (Fig. 11). Over time, the injury-induced progenitors both continue to multiply and migrate to the ONL (data not shown, see [35]).

Microglial cells invade the site of photoreceptor injury

In addition to dead and dying photoreceptors and proliferating cone progenitors, microglia are also present within the outer nuclear (and outer segment) layers (Fig. 2). Microglia, as identified by the 4C4 antibody, normally reside in the optic fiber layer, the plexiform layers, and among cells of the retinal pigment epithelium (RPE; Fig. 2a). At $24 \mathrm{~h}$ after light onset, the time of peak cell death, 4C4-positive microglial cells hypertrophy, become polymorphic, and migrate into the ONL (Fig. 2b). At $48 \mathrm{~h}$ after light onset, 4C4-positive microglia also invade the field of photoreceptor debris in the outer segment layer, between the ONL and the RPE. These microglia likely originate from those cells that normally reside within the RPE. The number of 4C4-positive microglia within the retina and among the dying photoreceptors increases until at least $72 \mathrm{~h}$ after light onset (Fig. 2c,d).
Fig. 5 Heat map of selected phototransduction genes that change over the course of the light injury. The majority of photoreceptor-specific genes decreased their levels of expression, consistent with photoreceptor loss due to cell death

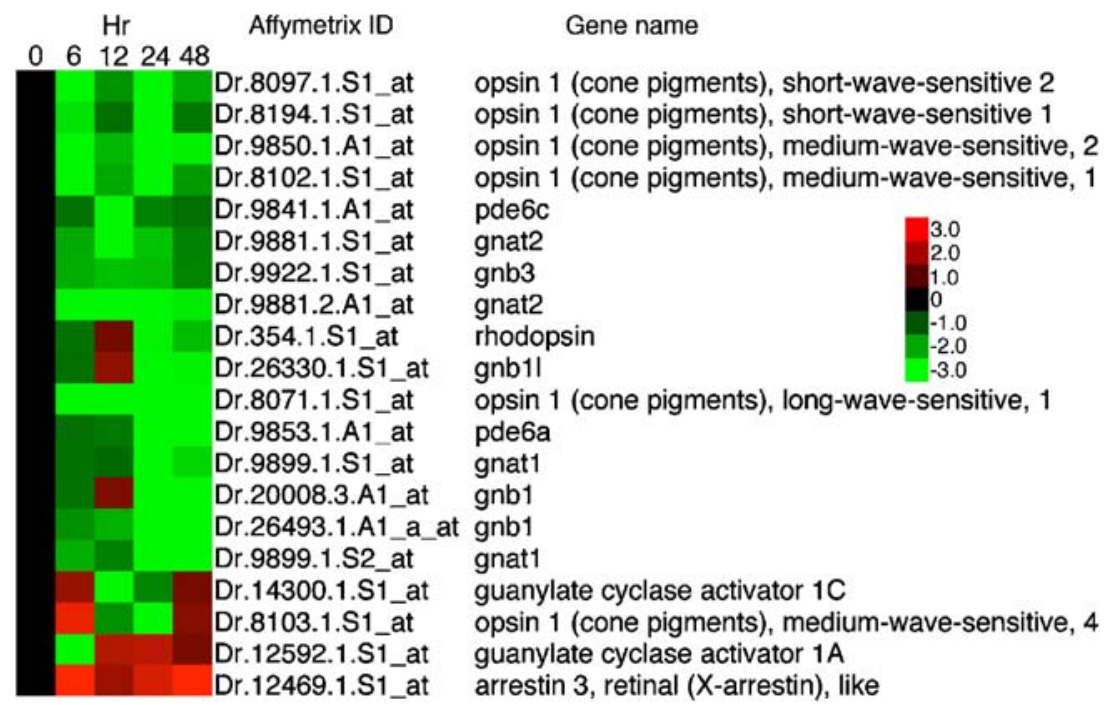


Fig. 6 Heat map of selected stress response genes that change in expression over the course of light injury. The majority of these genes showed marked increase in expression during the early phases of the light-induced injury

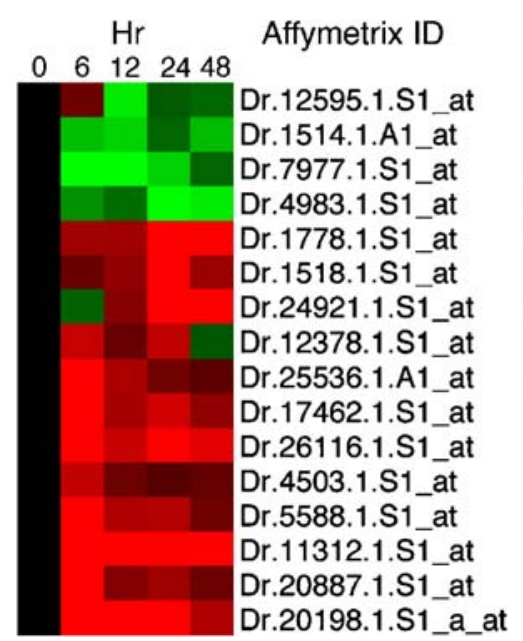

Gene name

heat shock factor 2

RAD51 homolog

glutathione peroxidase 1

glutathione peroxidase $4 a$ syndecan binding protein nudix

glutathione peroxidase $4 \mathrm{~b}$

heat shock protein 1

similar to heat shock protein HSP 90-alpha

hypoxia up-regulated 1

heat shock $70 \mathrm{kDa}$ protein 5

heat shock protein $9 \mathrm{~B}$

heat shock protein 4 , like

heat shock $10 \mathrm{kD}$ protein 1

DnaJ (Hsp40) homolog

heat shock cognate $70 \mathrm{kD}$ protein
Injury-induced changes in gene expression among cells of the outer nuclear layer

To identify injury-induced changes in gene expression, RNA was isolated from cells within the ONL of lightlesioned retinas using LCM (Fig. 3), and changes in gene expression between control retinas and retinas exposed to light for between 6 and $48 \mathrm{~h}$ were identified with oligonucleotide microarrays. Analysis of the microarrays across all time points showed that a total of 1,937 genes showed at least a twofold or greater change in signal intensity (gene expression) on the array (see Supplementary Material). Hierarchical clustering identified the temporal correlation for both increases and decreases in transcript levels (Fig. 4). We categorized the genes into functional groups based on the gene ontology descriptions provided by Affymetrix and by manual classification. Although only approximately $50 \%$ of these genes are annotated, we were able to assign a subset of the known genes to the cell types present in the ONL over the time points studied here.

Transcriptional changes predicted for injured photoreceptors

Based on our cellular analysis, and taking into account that at $0,6,12$, and $24 \mathrm{~h}$ after light onset photoreceptors are the numerically predominant cell type present in the ONL (Fig. 1), we infer that genes that change levels of expression compared to the 0 -h control during the first $24 \mathrm{~h}$ after light onset will largely be restricted to injured and dying photoreceptors (Fig. 5). To support this assertion, numerous known photoreceptor-specific genes showed decreased expression at early time points, including genes encoding opsins, pde6c (phosphodiesterase 6c), gnat (alpha transducin), and gnb (beta transducin; Fig. 5). Similarly, and as anticipated, at early time points, the expression of numerous
Fig. 7 Heat map of selected secreted proteins that change over the course of the light injury

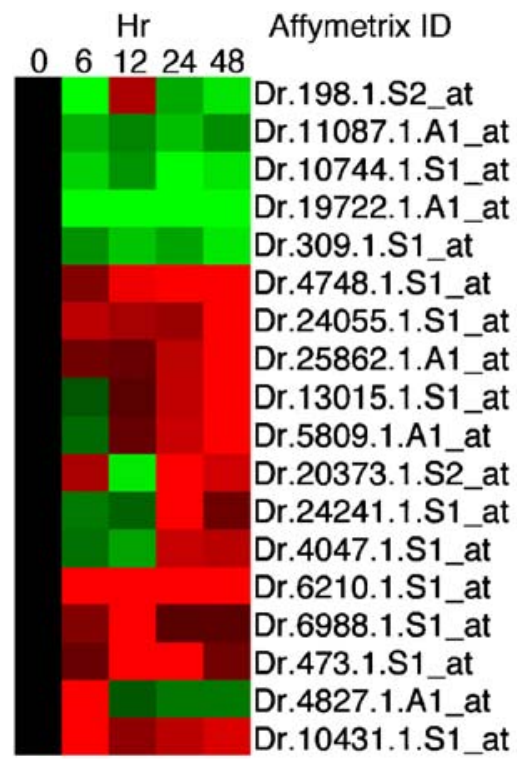

\section{Gene name}

$\begin{array}{ll}\text { similar to follistatin } & 3.0 \\ \text { secretogranin III } & 2.0 \\ \text { nerve growth factor, beta } & 1.0 \\ \text { insulin b } & -1.0 \\ \text { wnt8-like protein } 2 & -2.0 \\ \text { similar to granulin 1/granulin } 2 & -3.0 \\ \text { similar to CC chemokine-1 } & \text { galectin 9-like 1 } \\ \text { galectin 1-like } 2 & \\ \text { granulin a } \\ \text { midkine-related growth factor } \\ \text { midkine-related growth factor b } \\ \text { scyba } \\ \text { similar to cartilage acidic protein } 1 \\ \text { anti-dorsalizing morphogenic protein } \\ \text { inhibin, beta B } \\ \text { leukocyte cell derived chemotaxin } 1 \\ \text { connective tissue growth factor }\end{array}$


Fig. 8 Heat map of selected transcription factors that change over the course of light injury

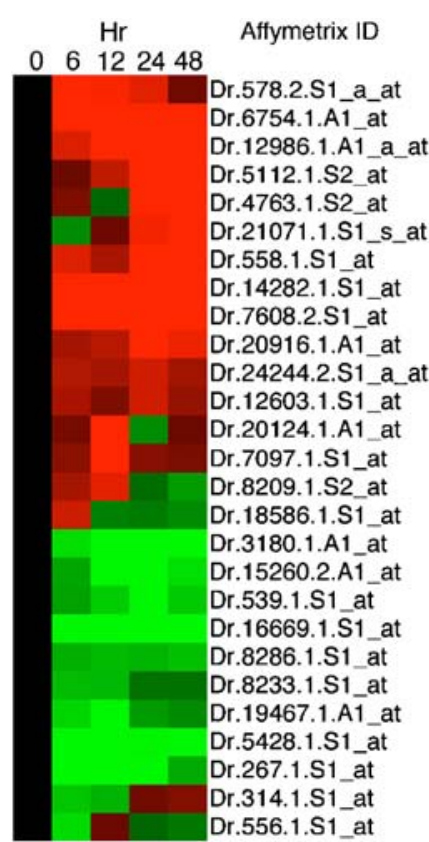

\section{Gene name}

tef (thyrotroph embryonic factor) per2 (period homolog 2)

fos (FBJ murine osteosarcoma viral oncogene homolog) sox 11b (SRY-box containing gene 11b) sox11a

pbx1a (pre-B-cell leukemia transcription factor 1a) vsx1 (visual system homeobox 1 protein) atf3 (activating transcription factor 3 ) jun isl2b (islet $2 b$ ) pax-6a (paired homeobox gene 6a) irx7 (iroquois homeobox protein 7) sox4a smad7 (MAD, mothers against decapentaplegic homolog 7) foxo5 (forkhead box 05)

tbx15 (T-box 15)

bhlhb2 (basic helix-loop-helix domain containing, class b, 2) reverbb2

rx2 (retinal homeobox gene 2)

nr2f1l (nuclear receptor subfamily 2 , group $F, 1$ ) lef1 (lymphocyte enhancer binding factor 1 ) tbx2b

hes6 (hairy and enhancer of split 6) id2a (inhibitor of DNA binding 2a) mef2c (myocyte enhancer factor 2c) ascl1a (achaete-scute complex-like 1a) neurod (neurogenic differentiation) stress response genes was dramatically increased (Fig. 6). Genes encoding secreted proteins also show changes at early time points, including insulin $b$, nerve growth factor $b$, and wnt-8-like-2 (Fig. 7). Although we are less certain regarding the identities of cells that express genes encoding these growth factors (see below), a first approximation is that some of these genes may be expressed by photoreceptors.
Transcriptional changes predicted to occur in cone progenitors and microglia

Following a similar logic, we predict that transcripts with levels that change at the 24- and 48-h time points compared to the control retinas will be expressed in cells that migrate to the ONL, cone progenitors, and microglia (see Figs. 1 and 2).
Fig. 9 Heat map of selected genes that showed a marked increase in transcription at the 48-h time point

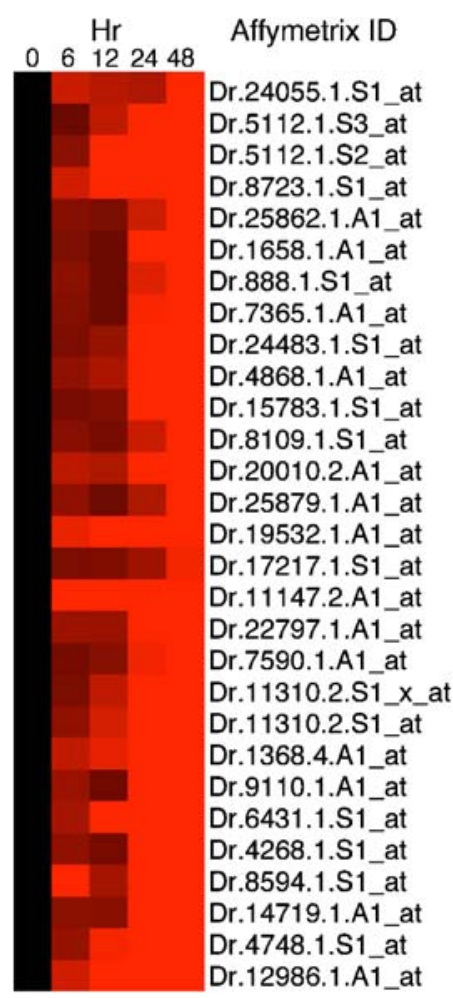

\section{Gene name}

similar to CC chemokine-1 sox11b (SRY-box containing gene 11b) sox11b similar to Thioredoxin (ATL-derived factor) galectin 9-like 1

Solute carrier family 4 , anion exchanger, member ATPase, $\mathrm{Na}+/ \mathrm{K}+$ transporting, alpha $1 \mathrm{a} .1$ wu:fj13h01

hypothetical protein LOC558116 Zgc:92630

lymphocyte cytosolic plastin 1 (I-plastin) tubulin, alpha 8 like

Transcribed locus

Transcribed locus

RAB32, member RAS oncogene family

Transcribed locus

Transcribed locus wu:fj68b05

tubulin, alpha 1 tubulin, alpha 1

--similar to Activating transcription factor 5 zgc:56537

wu:fb66f03

zgc: 100919

zgc:112199

similar to granulin 1/granulin 2

fos 

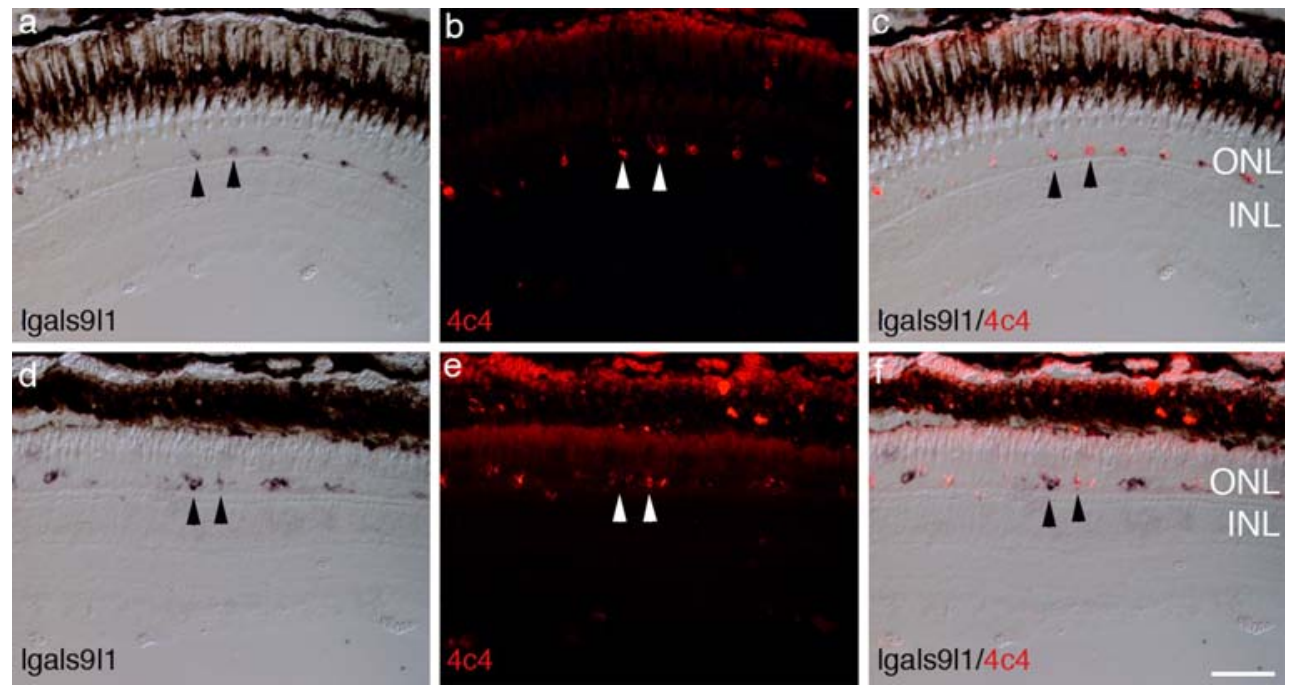

Fig. 10 Expression of Lgals $9 l 1$ mRNA in microglia in the $O N L$. Panels a-c illustrate a retinal section from an animal exposed to constant light for $12 \mathrm{~h}$, then labeled by in situ hybridization with probes against lgals $9 l 1 \mathrm{mRNA}$ (a), immunolabeled for microglia with 4C4 antibody (b). Panel $\mathbf{c}$ is the digital overlay of $\mathbf{a}$ and $\mathbf{b}$. Panels $\mathbf{d}-\mathbf{f}$ illustrate a retina section from an animal exposed to constant light for

Genes at the 24- and 48-h time points were among those that showed the greatest magnitude of change. Included in these genes are those encoding the transcription factors sox $11 b$, fos, jun, and pax6a (Figs. 8 and 9), and the secreted proteins, lymphocyte cytosolic plastin 1 (l-plastin, see [16]), CC chemokine, members of the galectin family, members of the progranulin family, and midkines $a$ and $b$ (Fig. 7).

From the genes encoding growth factors, three were selected to study further: two members of the galectin family, galectin-9 like-1 (lgals9l1) and galectin-1 like-2 (lgals 1l2), and progranulin-a (pgrna). In situ hybridization
$48 \mathrm{~h}$, then labeled by in situ hybridization with probes against lgals $9 l 1$ mRNA (d), immunolabeled for microglia with 4C4 antibody (e). Panel $\mathbf{f}$ is the digital overlay of $\mathbf{d}$ and e. Arrowheads indicate the same cells in $\mathbf{a}-\mathbf{c}$ and $\mathbf{d}-\mathbf{f}$. $O N L$, outer nuclear layer; $I N L$, inner nuclear layer. Scale bar $=50 \mu \mathrm{m}$

or immunocytochemistry were used both to independently validate the changes in the expression of these genes and to characterize their cellular patterns of expression. Lgals911 and Lgals 112 are members of a large family of secreted carbohydrate-binding proteins that bind $\beta$-galactosides on the surface of glycoproteins such as laminin, integrins, osteopontin, and fibronectin [5]. Galectins are believed to mediate interactions between cells and the extracellular matrix [27]. In situ hybridization showed that lgals9l1 is expressed by a sparse population of cells confined to a narrow strata at the interface of the ONL and outer
Fig. 11 Lgals112 protein is expressed by proliferating Müller glial stem cells and their progeny and microglia in the $O N L$ of injured retina. Panels a-c illustrate a retinal section from a transgenic ( $g$ fap: GFP) ${ }^{\text {mi2001 }}$ fish exposed to constant light for $48 \mathrm{~h}$ followed by immunolabeling for Lgals112 (a) and GFP (b). Panel $\mathbf{c}$ is a digital overlay of $\mathbf{a}$ and $\mathbf{b}$. Panels d-f illustrate a retinal section from an animal exposed to constant light for $48 \mathrm{~h}$ and immunolabeled for Lgals112 (d) and $4 \mathrm{C} 4$ (e). Panel $\mathbf{f}$ is a digital overlay of $\mathbf{d}$ and e. Arrowheads indicate the same cells in $\mathbf{a}-\mathbf{c}$ and $\mathbf{d}-\mathbf{f}$. Scale bar $=50 \mu \mathrm{m}$. $O N L$, outer nuclear layer; $I N L$, inner nuclear layer
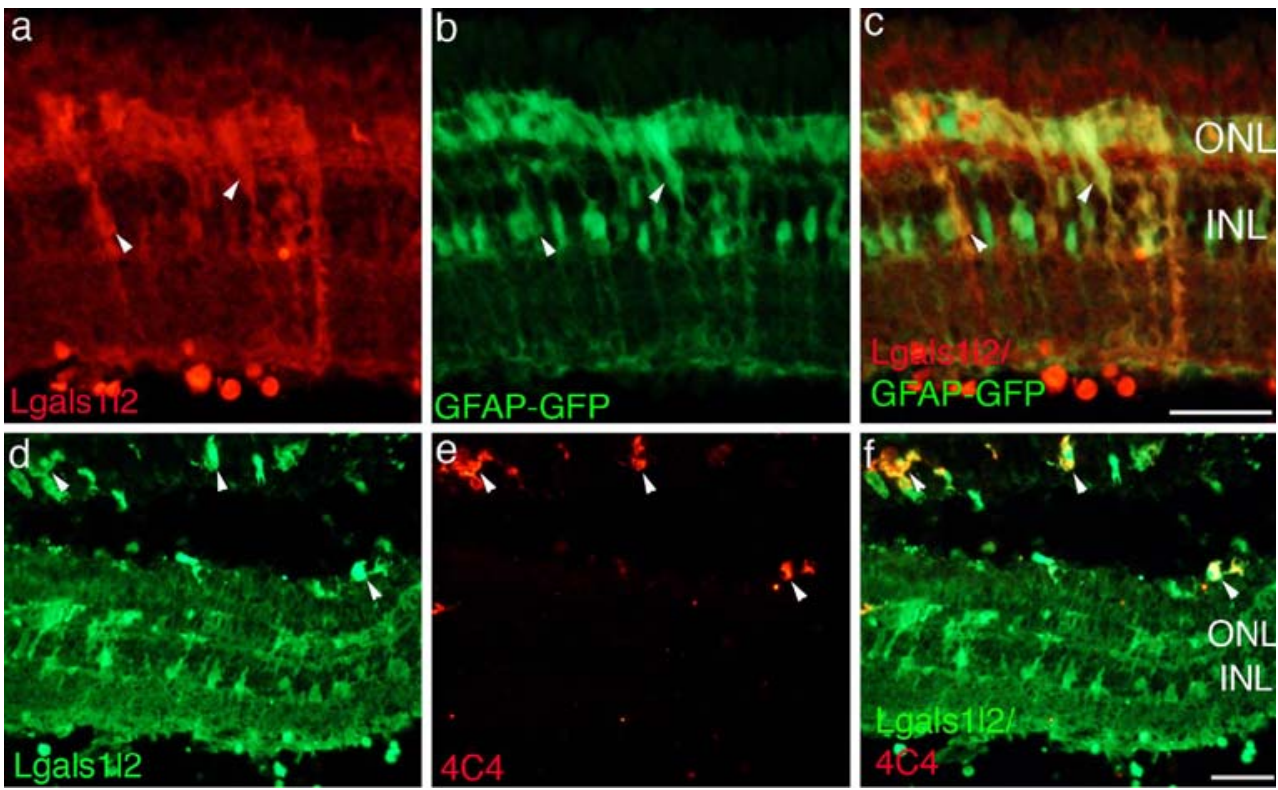

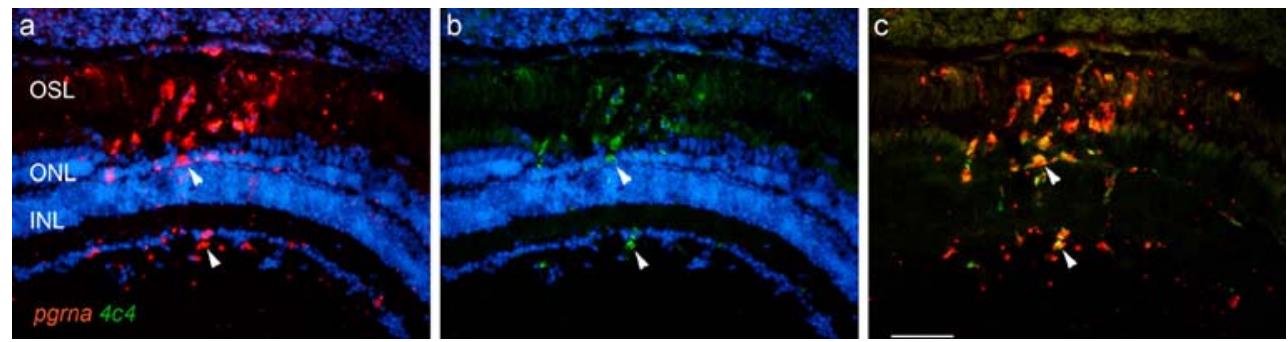

Fig. 12 Progranulin- $a$ is expressed by microglia at the site of photoreceptor injury. Panel a illustrates an in situ hybridization showing pgrna at the site of photoreceptor injury. Panel $\mathbf{b}$ is the same section as in a but immunostained with the $4 \mathrm{c} 4$ antibody. Panel $\mathbf{c}$ is the

plexiform layer, a location that is suggestive of microglia (Fig. 10; see [37]). This inference was confirmed by combining in situ hybridization with immunolabeling with the microglia-specific antibody, 4c4 (Fig. 10). In contrast, immunostaining with antibodies against Lgals112 labeled both radial columns of cells spanning the INL, suggestive of Müller glia and/or cone progenitors, and microglia. Retinal sections taken from the $\operatorname{Tg}(g f a p: G F P)^{\text {mi2001 }}$ line and immunostained with the Lgals112 antibodies confirmed that cells immunopositive for Lgals112 originate from Müller glia (Fig. 11a-c), and immunostaining with $4 \mathrm{c} 4$ identified GFP-negative, Lgals112-positive microglia.

Fig. 13 Heat map highlighting selected genes that increased expression at the $12-\mathrm{h}$ time point nuclear layer; $I N L$, inner nuclear layer microglia (Fig. 12b,c).

\section{Affymetrix ID Gene name}

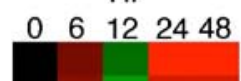

digital overlay of panels a and $\mathbf{b}$. Arrowheads identify the same cells in a-c. Scale bar $=50 \mu \mathrm{m}$. OSL, outer segment layer; $O N L$, outer

Progranulin-a (a.k.a., granulin-epithelin precursor, proepithelin, PC cell-derived growth factor, acrogranin) is one of the five granulin paralogs expressed in zebra fish [4] that encode proteins with multiple functions, including mediating tissue repair [15]. In situ hybridization with probes encoding pgrna labeled cells within the retina that are in close association with the lesioned photoreceptors and, most prominently, cells within the ONL and the overlying outer segment layer (Fig. 12a). Combining in situ hybridization with immunostaining with the $4 \mathrm{c} 4$ antibody showed that pgrna is expressed exclusively by

Dr.10901.1.S1_at Dr.3373.1.S1_at Dr.24178.1.S1_at Dr.11133.1.A1_at Dr.21904.1.A1_at Dr.22720.1.A1_at Dr.20373.1.S2_at Dr.16206.1.A1_at Dr.13799.1.S1_x_at Dr.25102.1.A1_at Dr.26033.1.A1_at Dr.12310.1.A1_at Dr.8629.1.A1_at Dr.23416.1.A1_at Dr.10598.1.S1_at Dr.21601.1.A1_at Dr.25063.1.S1_at Dr.10405.1.S1_at Dr.12569.1.S1_at Dr.4763.1.S2_at Dr.4763.3.S1_at Dr.22000.1.A1_at Dr.22685.1.A1_at Dr.5623.1.S1_at Dr.5258.1.A1_at Dr.4219.1.S1_at Dr.4106.1.A1_at Dr.21393.1.A1 at Dr.10336.1.S1_at Dr.12451.2.A1_at Dr.906.1.S1_at Dr.5820.1.S1_at

\author{
Wiskott-Aldrich syndrome, like \\ collagen type XVIII, alpha 1 \\ Transcribed locus \\ dihydropyrimidinase-like $5 \mathrm{~b}$ \\ wu:fc79g11 \\ Transcribed locus \\ midkine-related growth factor \\ similar to embryonic transglutaminase \\ Hypothetical protein LOC407696 \\ transducer of ERBB2, 1b \\ Transcribed locus \\ Transcribed locus \\ wu:fc06b04 \\ Wu:fc92f06 \\ zgc:55316 \\ wu:fc $25 \mathrm{c} 08$
}

Transcribed locus

carbonic anhydrase II

Kruppel-like factor d

SRY-box containing gene 11a

SRY-box containing gene 11a

wu:fd02b11

zgc:91929

zgc:55345

wu:fc51h06

wu:fb36b08

wu:fc12g11

dopachrome tautomerase

zgc:73213

Activated leukocyte cell adhesion molecule similar to cathepsin L preproprotein similar to creatine kinase, brain 
Transcriptional changes at $12 \mathrm{~h}$ after light onset may identify genes regulated by light or the circadian rhythm

Fortuitously, the design of our experiment also allowed us to identify transcriptional changes that are likely regulated either by light or the circadian rhythm. Cells harvested at 0 (control), 24-, and 48-h time points were collected in the morning shortly after the room lights were switched on, whereas the 12-h time points were collected shortly before the room lights were switched off. Therefore, genes that are differentially expressed at 12- vs. 0-, 24-, or 48-h time points may encode proteins that are synthesized in response to light or regulated by the circadian rhythm [13]. As evidence for this, we detected numerous genes that were differentially expressed at the 12-h time point (Figs. 13 and 14).

\section{Discussion}

The dataset described in this report provides the vision community with a resource to mine for transcriptional changes that regulate the first stages of photoreceptor regeneration, from death to the specification of photoreceptor progenitors. Early transcriptional changes were observed in genes likely expressed by injured photoreceptors. Later transcriptional changes allowed us to identify novel genes expressed in Müller glia and cone progenitors, which give rise to regenerated photoreceptors, and microglia, which may also play a crucial role in photoreceptor regeneration. More broadly, this dataset provides information about the molecular mechanisms that regulate injuryinduced neurogenesis in an adult nervous system and thus has utility for neuroscience in general.

By combining LCM of the ONL with a time course of injury-induced gene expression, we were able nominally to assign genes to each of the three cell types the cellular analysis identified within the lesioned ONL. We predicted that changes in gene expression that occur during the first $24 \mathrm{~h}$ are largely attributable to the injured photoreceptors. Thus, as anticipated, we observed transcriptional changes in known injury-induced genes at the early time points, including the heat shock proteins. Similarly, we detected a
Fig. 14 Heat map highlighting selected genes that decreased in expression at the 12 -h time point

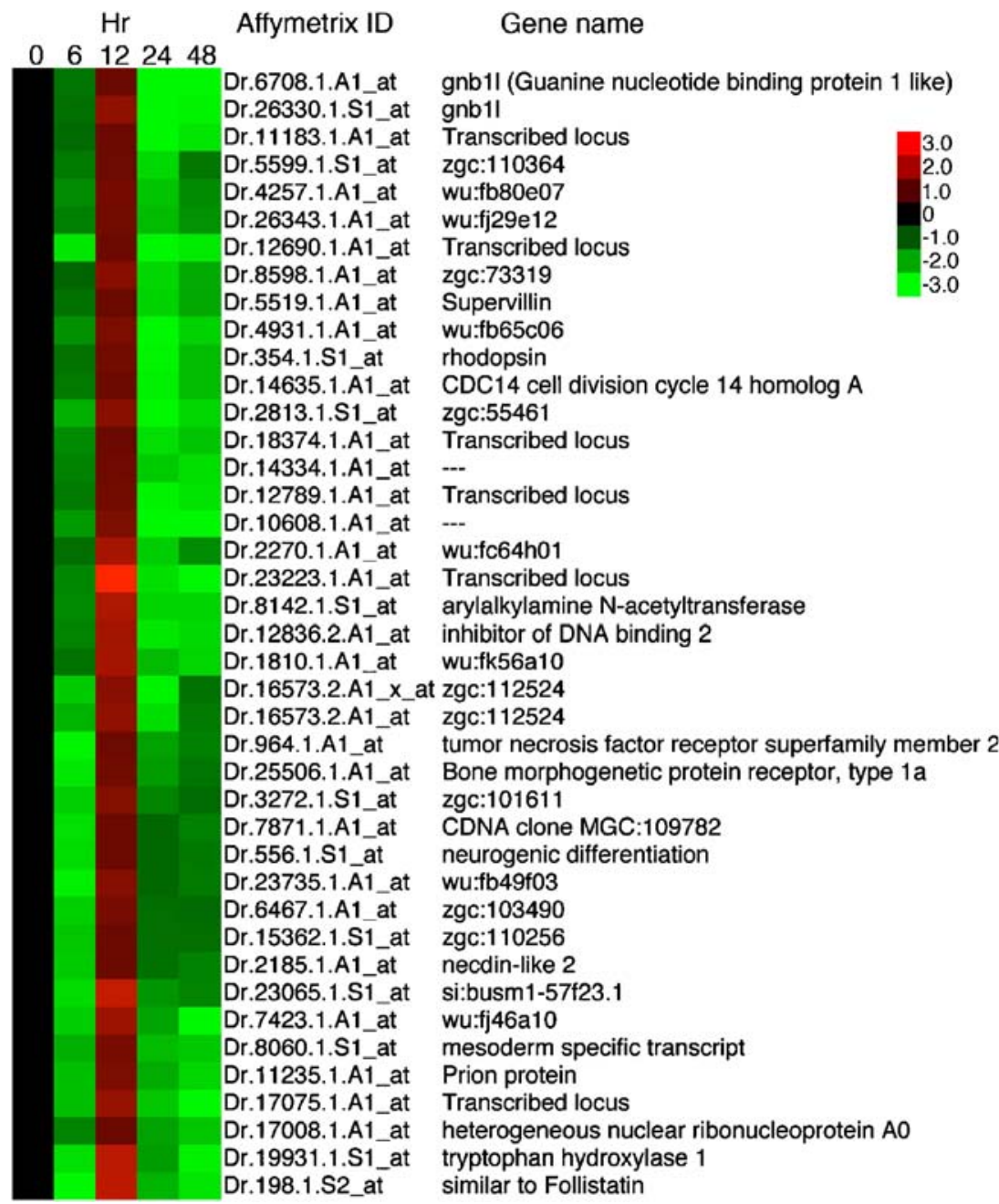


decrease in photoreceptor-specific genes, including genes encoding opsins and phototransduction proteins. The decrease in the expression of these genes is most easily explained by the apoptotic loss of photoreceptors. Nonetheless, the observed decrease in photoreceptor-specific genes serves to validate the logic behind our approach to assign early gene changes to photoreceptors. In the mammalian retina, injury to photoreceptors results in increased transcription of endothelin2, which encodes a secreted protein that signals through endothelin B receptors on Müller glia [28]. We anticipate that similar signaling events occur in the regenerating retina of teleosts (although no changes in endothelin 1 or 2 were observed), and genes encoding molecules that signal from photoreceptors to Müller glia are represented in the dataset. One category of genes worth investigating further are the stress response genes. Recent studies have suggested that heat shock proteins may be secreted [8] and thus may serve as extracellular signaling molecules during the early events of photoreceptor regeneration.

In addition, we anticipated identifying known and novel genes expressed by cone progenitors in the ONL at the 48-h time point. It should be noted, however, that the changes in the expression of these genes, and in contrast to the loss of photoreceptors, are most easily explained by the accretion of cone progenitors migrating from the INL to the ONL. Nonetheless, our approach allowed us to identify putative cone-progenitor-specific genes, and these data will provide insights into the molecular mechanisms that govern the terminal steps of cone specification and differentiation. In support of this hypothesis, we detected an increase in the expression of lgals 112 , which encodes a secreted protein, and demonstrated that Lgals 112 is expressed by putative cone progenitors (and microglia and Müller glia).

Based on our cellular analysis, we also anticipated identifying genes expressed by reactive microglia that migrate to the damaged ONL. Microglia are a little studied cell type but one whose importance in various developmental and disease states is becoming recognized [14, 24]. Of note, each of the genes encoding the secreted proteins lgals 112 , lgals $9 l 1$, and prgna is expressed by resident microglia after injury to photoreceptors. Microglia are phagocytic cells that can secrete both proinflammatory and anti-inflammatory signals to either inhibit or promote neuronal repair and regeneration [14]. Therefore, microglial-derived secreted signals are likely important regulators of cone photoreceptor regeneration. Functional evaluation of microglial-specific genes should provide further insights into the mechanisms that regulate photoreceptor (and neuronal) regeneration.

Gene array technologies have been used previously to profile transcriptional changes in zebra fish during regeneration of the fin [32], heart [22], and retina [6, 21]. Recently, LCM and gene arrays were combined to identify transcriptional changes in retinal ganglion cells during axonal regeneration [34]. Of note, some of the gene coding for secreted factors identified in our dataset are also upregulated during heart and fin regeneration [22, 32]. The injury-induced upregulation of these genes suggest that in zebra fish common molecular mechanisms may regulate all regenerative processes. However, further comparisons of datasets from zebra fish arrays should also reveal molecular signatures that are specific to retinal stem cells and the regeneration of cone photoreceptors. Finally, although the annotation of the zebra fish genome does not yet match that for mammals, comparing transcriptional changes in the injured and regenerating retina of zebra fish with similar studies conducted in mammals $[7,28]$ may begin to identify molecular mechanisms that are essential to control the fates of stem cells in the mammalian retina and brain.

Acknowledgements We thank Drs. Pamela Raymond for the transgenic (gfap:GFP) ${ }^{\mathrm{mi2} 2001}$ reporter fish and the 4C4 antibody, Geraldo Vasta for the anti-zebra-fish Galectin-1-like-2 antibody and Hugh Bennett for the progranulin- $a$ cDNA. We also thank Dr. David Reed, Matthew Brooks, and Ritu Khanna for their help in conducting and analyzing the microarray experiments and Erica Dawsey and Laura Kakuk-Atkins for technical assistance. This work was supported by the National Institutes of Health Grants R01-EY007060 and P30EY07003 (PFH) and T32-EY13934 (S.E.L.C.) and Research to Prevent Blindness. S.E.L.C. was also supported by a postdoctoral fellowship from the Foundation Fighting Blindness-Canada.

Open Access This article is distributed under the terms of the Creative Commons Attribution Noncommercial License which permits any noncommercial use, distribution, and reproduction in any medium, provided the original author(s) and source are credited.

\section{References}

1. Benjamini Y, Yekutieli DJ. Am Stat Assoc 2005;100:71-80.

2. Bernardos RL, Raymond PA. GFAP transgenic zebra fish. Gene Expr Patterns 2006;6:1007-13.

3. Bernardos RL, Barthel LK, Meyers JR, Raymond PA. Late-stage neuronal progenitors in the retina are radial Müller glia that function as retinal stem cells. J Neurosci 2007;27:7028-40.

4. Cadieux B, Chitramuthu BP, Baranowski D, Bennett HP. The zebra fish progranulin gene family and antisense transcripts. BMC Genomics 2005;6:156.

5. Camby I, Le Mercier M, Lefranc F, Kiss R. Galectin-1: a small protein with major functions. Glycobiology 2006;16:137R-157R.

6. Cameron DA, Gentile KL, Middleton FA, Yurco P. Gene expression profiles of intact and regenerating zebra fish retina. Mol Vis 2005;11:775-91.

7. Chen L, Wu W, Dentchev T, Zeng Y, Wang J, Tsui I, Tobias JW, Bennett J, Baldwin D, Dunaief JL. Light damage induced changes in mouse retinal gene expression. Exp Eye Res 2004;79:239-47.

8. Eustace BK, Sakurai T, Stewart JK, Yimlamai D, Unger C, Zehetmeier C, Lain B, Torella C, Henning SW, Beste G, Scroggins BT, Neckers L, Ilag LL, Jay DG. Functional proteomic screens reveal an essential extracellular role for hsp90 alpha in cancer cell invasiveness. Nat Cell Biol 2004;6:507-14.

9. Fausett BV, Goldman D. A role for alphal tubulin-expressing Müller glia in regeneration of the injured zebra fish retina. J Neurosci 2006;26:6303-13. 
10. Fausett BV, Gumerson JD, Goldman D. The proneural basic helix-loop-helix gene asclla is required for retina regeneration. $\mathrm{J}$ Neurosci 2008;28:1109-17.

11. Fimbel SM, Montgomery JE, Burket CT, Hyde DR. Regeneration of inner retinal neurons after intravitreal injection of ouabain in zebra fish. J Neurosci 2007;27:1712-24.

12. Fischer AJ, Reh TA. Müller glia are a potential source of neural regeneration in the postnatal chicken retina. Nat Neurosci 2001;4:247-52.

13. Green CB, Besharse JC. Retinal circadian clocks and control of retinal physiology. J Biol Rhythms 2004;19:91-102.

14. Hanisch UK, Kettenmann H. Microglia: active sensor and versatile effector cells in the normal and pathologic brain. Nat Neurosci 2007;10:1387-94.

15. He Z, Ong CH, Halper J, Bateman A. Progranulin is a mediator of the wound response. Nat. Med. 2003;9:225-9.

16. Herbomel P, Thisse B, Thisse C. Zebra fish early macrophages colonize cephalic mesenchyme and developing brain, retina, and epidermis through a M-CSF receptor-dependent invasive process. Dev Biol 2001;238:274-88.

17. Hero AO, Fleury G, Mears AJ, Swaroop A. Multicriteria gene screening for analysis of differential expression with DNA microarrays. EURASIP JASP 2004;2004:43-52.

18. Hitchcock PF. Tracer coupling among regenerated amacrine cells in the retina of the goldfish. Vis Neurosci 1997;14:463-72.

19. Hitchcock P, Kakuk-Atkins L. The basic helix-loop-helix transcription factor neuroD is expressed in the rod lineage of the teleost retina. J Comp Neurol 2004;477:108-17.

20. Hitchcock PF, Lindsey Myhr KJ, Easter SS Jr, Mangione-Smith $\mathrm{R}$, Jones DD. Local regeneration in the retina of the goldfish. $\mathrm{J}$ Neurobiol 1992;23:187-203.

21. Kassen SC, Ramanan V, Montgomery JE, Burket CT, Liu CG, Vihtelic TS, Hyde DR. Time course analysis of gene expression during light-induced photoreceptor cell death and regeneration in albino zebra fish. Dev Neurobiol 2007;67:1009-31.

22. Lien CL, Schebesta M, Makino S, Weber GJ, Keating MT. Gene expression analysis of zebra fish heart regeneration. PLoS Biol 2006;4:e260.

23. Mader MM, Cameron DA. Photoreceptor differentiation during retinal development, growth, and regeneration in a metamorphic vertebrate. J Neurosci 2004;24:11463-72.

24. Mallat M, Marín-Teva JL, Chéret C. Phagocytosis in the developing CNS: more than clearing the corpses. Curr Opin Neurobiol 2005; 15:101-7.
25. Ooto S, Akagi T, Kageyama R, Akita J, Mandai M, Honda Y, Takahashi M. Potential for neural regeneration after neurotoxic injury in the adult mammalian retina. Proc Natl Acad Sci U S A 2004;101:13654-9.

26. Osakada F, Ooto S, Akagi T, Mandai M, Akaike A, Takahashi M. Wnt signaling promotes regeneration in the retina of adult mammals. J Neurosci 2007;27:4210-9.

27. Rabinovich GA, Toscano MA, Jackson SS, Vasta GR. Functions of cell surface galectin-glycoprotein lattices. Curr. Opin. Struct. Biol 2007; 17:513-20.

28. Rattner A, Nathans J. The genomic response to retinal disease and injury: evidence for endothelin signaling from photoreceptors to glia. J Neurosci 2005;25:4540-9.

29. Raymond PA. Retinal regeneration in teleost fish. Ciba Found Symp 1991;160:171-86.

30. Raymond PA, Reifler MJ, Rivlin PK. Regeneration of goldfish retina: rod precursors are a likely source of regenerated cells. J Neurobiol 1988;19:431-63.

31. Raymond PA, Barthel LK, Bernardos RL, Perkowski JJ. Molecular characterization of retinal stem cells and their niches in adult zebra fish. BMC Dev Biol 2006;6:36.

32. Schebesta M, Lien CL, Engel FB, Keating MT. Transcriptional profiling of caudal fin regeneration in zebra fish. Scientific World Journal 2006;6(Suppl 1):38-54.

33. Thummel R, Kassen SC, Montgomery JE, Enright JM, Hyde DR. Inhibition of Müller glial cell division blocks regeneration of the light-damaged zebra fish retina. Dev Neurobiol 2008;68:392-408.

34. Veldman MB, Bemben MA, Thompson RC, Goldman D. Gene expression analysis of zebra fish retinal ganglion cells during optic nerve regeneration identifies KLF6a and KLF7a as important regulators of axon regeneration. Dev Biol 2007;312:596-612.

35. Vihtelic TS, Hyde DR. Light-induced rod and cone cell death and regeneration in the adult albino zebra fish (Danio rerio) retina. J Neurobiol 2000;44:289-307.

36. Vihtelic TS, Soverly JE, Kassen SC, Hyde DR. Retinal regional differences in photoreceptor cell death and regeneration in lightlesioned albino zebra fish. Exp Eye Res 2006;82:558-75.

37. Wagner EC, Raymond PA. Müller glial cells of the goldfish retina are phagocytic in vitro but not in vivo. Exp Eye Res 1991;53: $583-9$.

38. Wu DM, Schneiderman T, Burgett J, Gokhale P, Barthel L, Raymond PA. Cones regenerate from retinal stem cells sequestered in the inner nuclear layer of adult goldfish retina. Invest Ophthalmol Vis Sci 2001;42:2115-24. 